Review

\title{
Induction of Atherosclerosis by Human Chylomicron Remnants:
}

\section{A Hypothesis}

\author{
Monica G. Wilhelm and Allen D. Cooper \\ Stanford University School of Medicine, Research Institute, Palo Alto Medical Foundation, Palo Alto, CA, USA.
}

\begin{abstract}
Epidemiologic studies have provided support for the association between delayed remnant removal and premature atherosclerosis. Triglyceride-rich particles such as chylomicrons and chylomicron remnants that carry dietary derived fats, may play a role in the early stages of developing arteriosclerosis. Currently research focuses on these lipoprotein classes seeking distinguishing factors that causes some lipoproteins to be atherogenic while others are not. Such lipoproteins could be involved in atherogenesis directly or indirectly. Direct involvement occurs by interaction of triglyceride-rich particles with the arterial wall, possibly affecting the artery wall by oxidative stress, direct endothelial toxicity by constituents such as lysophosphatidylcholine or oxysterols, induction of prothrombotic changes, stimulation of endothelial expression of cell adhesion molecules and direct interaction with circulating blood cells. Indirect involvement refers to the influence of triglyceride-rich lipoproteins on other lipoproteins on the composition of low density lipoprotein (LDL) and high density lipoprotein (HDL) particles. We propose that in individuals with delayed removal of chylomicron remnants, the prolonged exposure of areas of endothelium that have been partially activated by turbulent flow, to specific components of the remnants, results in the endothelial cells becoming further activated and able to bind monocytes. During or shortly after the transcytosis to the intima and transformation of monocytes to macrophages, the macrophages become engorged with remnant derived lipids and form the nidus of a fatty streak. J Atheroscler Thromb, 2003; 10: 132-139.
\end{abstract}

Key words: Chylomicron remnants, Triglyceride-rich lipoproteins, Atherosclerosis, Artery

\section{Introduction}

After the causal relationship between plasma cholesterol and coronary artery disease became clear, research focused on the role of triglycerides (TG) in the development of arteriosclerosis. It became apparent, that the atherogenic role of triglycerides might be different from that of cholesterol. While "more is worse" with plasma cholesterol, more is not always worse with plasma tri-

Address for correspondence: Allen D. Cooper, Research Institute, Palo Medical Foundation, Ames Research Building, 795 El Camino Real, Palo Alto, CA 94301, USA.

E-mail: adc@stanford.edu

Received February 12, 2003.

Accepted February 19, 2003. glycerides in terms of coronary artery disease risk (1), and while the cholesterol level does not undergo postprandial changes, triglyceride levels do. An important role for postprandial triglycerides in arteriosclerosis was proposed by Zilversmit (2) in 1979. Since triglycerides are associated with free and esterified cholesterol, apoproteins and phospholipids as lipoprotein particles, research now focuses on triglyceride-rich lipoprotein classes with the aim of finding distinguishing factors that

Abbreviations: TG, triglyceride; TRL, triglyceride-rich proteins; CM, chylomicrons; VLDL, very low density lipoprotein; LDL, low density lipoprotein; HDL, high density lipoprotein; PAF-AH, platelet-activating factor acetylhydrolase; CAMs, cell adhesion molecules. 
causes some lipoproteins to be atherogenic while others are not. According to current postulation triglycerides may be involved in atherogenesis directly or indirectly. Direct involvement refers to an actual participation of triglyceride-rich lipoproteins (TRL) in the process of atherogenesis, while indirect involvement refers to the influence of TRL on other lipoproteins or substances, in some cases not necessarily related to lipid metabolism, which are then somehow made more atherogenic. This review focuses on the role of chylomicron remnants in atherogenesis. First, we review the evidence for the direct involvement and second we review the influence of chylomicrons (CM) and CM remnants on atherogenesis via their influence on low density lipoprotein (LDL) and high density lipoprotein (HDL).

\section{Exogenous and Endogenous Lipid Pathways}

Atherogenic lipoproteins can be derived from both the exogenous and endogenous pathways. While all lipoproteins carry triglycerides, some are considerably more rich in triglycerides than others. TGRL include CM and very low density lipoproteins (VLDL) as well as remnants of CM and VLDL which contain significant amounts of both cholesterol and triglycerides. LDL and HDL are cholesterol rich and triglyceride poor. Lipoprotein levels are generally measured in the fasting state; however, in industrialized countries, where three meals per day are consumed, most of the day is spent in the postprandial period. Thus CM and their remnants, the lipoproteins that carry diet derived triglycerides, can play an important role in atherogenesis. Dietary fatty acids are taken up into the intestinal cell and become re-esterified to triglycerides. There apolipoprotein B-48, the essential structural apolipoprotein of $\mathrm{CM}$ combine with triacylglycerol to form CM. Phospholipids and cholesterol and perhaps other proteins are added during this assembly process. Via the lymph, CM reach the plasma and the bulk of their triglycerides are hydrolyzed by lipoprotein lipase, the most important enzyme in plasma triglyceride catabolism. Lipoprotein lipase is bound to proteoglycans on the endothelial cells that line blood vessels in adipose tissue and muscle. In the presence of the co-factor apolipoprotein C-II, lipoprotein lipase can hydrolyze the triglycerides in chylomicrons. After sufficient hydrolysis, the remnants are released from the endothelium and reach the liver where apo $\mathrm{E}$ or the particle binds to the LDL receptor and the LDL receptor-related protein. A small amount of $\mathrm{CM}$ and remnants are rapidly taken up by cells of the reticuloendothelial system such as bone marrow and spleen $(3,4)$ and also by peripheral muscle and adipose tissue (5). Even though these particles are called triglyceride-rich and do in fact deliver abount $20 \%$ of dietary fat to the liver it is important to note, that on a per particle basis theses particles carry a large amount of cholesterol.

The main factors responsible for the clearance of triglyceride-rich lipoproteins are lipoprotein lipase, apolipoprotein E and the receptors. Defects in any of these proteins will lead to remnant accumulation and thereby to mixed hyperlipidemias (6). VLDL are hydrolyzed by endothelial bound lipoprotein lipase. Their remnant lipoproteins, often called IDL can be taken up by the liver using the LDL receptor and perhaps the LDL receptor related protein.

\section{Mechanism of Disease}

The accumulation of lipids within the arterial wall has been recognized as a key factor in atherogenesis for a long time and this led to the realization that the plasma cholesterol concentration is a major risk factor. Induced abnormalities of lipid and particularly cholesterol metabolism are sufficient to stimulate atherosclerosis. Monogenic disorders such as familial hypercholesterolemia, apoE or apo(a) genotype, Tangier disease, $\beta$-sitosterolemia and polymorphism in the hepatic lipase gene are all associated with accelerated atherosclerosis (7). However, it is now clear that the pathogenesis is much more complex. Currently a good deal of focus is on the role of inflammation in the genesis of the disease (8). The lesions of atherosclerosis occur principally in large and medium-sized elastic and muscular arteries and can lead to ischemia of the heart, brain, or extremities, resulting eventually in infarction of the organ supplied by the blood vessels. Lesions may be present throughout a person's lifetime. In fact, the earliest type of lesions, the so-called fatty streak is common in children and young adults and is largely an inflammatory lesion, consisting only of lipid laden monocyte-derived macrophages and T lymphocytes (9).

\section{Factors that induce and promote inflammation or atherogenesis}

The response-to-injury hypothesis of atherosclerosis initially proposed that endothelial denudation was the first step in atherosclerosis $(10,11)$. The most recent version of this hypothesis emphasizes endothelial dysfunction rather than denudation (9), another formulation emphasizes the presence of lipoproteins in the artery wall (12). Whichever process is at work, each characteristic lesion of atherosclerosis represents a different stage in a chronic inflammatory process in the artery. If unabated and excessive, this process will result in an advanced, complicated lesion. Proposed causes of endothelial dysfunction leading to atherosclerosis include elevated and modified LDL, free radicals caused by cigarette smoking, hypertension, diabetes mellitus, genetic alterations, elevated plasma homocysteine concentrations, infectious microorganisms 
such as herpes viruses or Chlamydia pneumoniae and combinations of these or other factors. Regardless of the cause of endothelial dysfunction, atherosclerosis is a highly characteristic response of specific sites in specific arteries (9). Injury of the arteries caused by these various factors results in endothelial dysfunction to which the arteries react with compensatory responses. Following injuries caused by these divergent factors, a common response is the increase of the adhesiveness of the endothelium with respect to leukocytes or platelets. There are also often changes of the endothelial permeability. In addition, injury also induces the endothelium to have procoagulant instead of anti-coagulant properties and to secrete vasoactive molecules, cytokines, and growth factors. If the inflammatory response does not effectively neutralize or remove the offending agents, it can continue indefinitely. In doing so, the inflammatory response stimulates migration and proliferation of smooth-muscle cells that become intermixed with the area of inflammation to form an intermediate lesion. If these responses continue unabated, they can thicken the artery wall, which compensates by gradual dilation, so that up to a point the lumen remains unaltered, a phenomenon termed "remodeling" (13). The response to injury is mediated by monocyte-derived macrophages and specific subtypes of $T$ lymphocytes at every stage of the disease $(14,15)$. With continuing inflammation the numbers of macrophages and lymphocytes increases and both cell types emigrate from the blood and multiply within the lesion. If these cells are activated, hydrolytic enzymes like metaloproteases are released which can induce further damage and eventually lead to focal necrosis (16-18). Thus cycles of accumulation of mononuclear cells, migration and proliferation of smooth-muscle cells, and formation of fibrous tissue lead to further enlargement and restructuring of the lesion, so that it becomes covered by a fibrous cap that overlies a core of lipid and necrotic tissue, a so-called advanced, complicated lesion. At some point, the artery can no longer compensate by dilation, the lesion may then intrude into the lumen and alter the flow of blood (9).

\section{Direct involvement of chylomicron remnants in atherogenesis Interaction with the arterial wall}

The size and density of triglyceride-righ lipoproteins is important because it is generally believed that the larger and more buoyant particles, particularly $\mathrm{CM}$, have little or no atherogenic potential. Newly made TRL, however, lose triglycerides and become atherogenic (2). Lipoprotein-arterial wall interactions in humans and in animal models have been studied by several groups (19-22) and have been reviewed (23-25). The reported results are that the influx of lipoproteins into the arterial wall depends primarily on the size of the lipoprotein particle, the concentration and the permeability of the arterial wall, and the blood pressure. Large CM are excluded from entry into the arterial intima (26) and the transfer of lipoproteins across the endothelium appears to be a non-receptormediated process of transcytosis or molecular sieving. Despite their size, chylomicron remnants and VLDL have been shown to penetrate arterial tissue, although, at a lower rate than $\mathrm{LDL}$ or $\operatorname{HDL}(27,28)$. Studies on lipoprotein efflux showed that after entry into the intima, triglyceridecontaining lipoproteins can become trapped (25) and it has been postulated by Williams and Tabas (12) that subendothelial retention of atherogenic lipoproteins may be an initiating pathogenic event in atherogenesis. Once in the intima larger lipoproteins are retained there longer than small lipoproteins, which may enhance the atherogenicity of triglyceride-rich lipoproteins. There, they interact with extracellular matrix, enhancing their entrapment and their potential interactions with intima cells to induce foam cell formation (29). Each triglyceriderich particle that enters the intima delivers 5 to 20 fold more cholesterol than does each LDL particle and is therefore potentially more atherogenic on a particle basis.

A review by Steiner (30) suggests that the importance of triglyceride-rich lipoproteins VLDL, CM and their remnant is in the development of arteriosclerosis may depend on the stage of the disease and postulates that these lipoproteins are only involved in the early stages of atherogenesis. Certain triglyceride-rich lipoproteins have been shown to be the only native, nonmodified lipoproteins that can cause rapid, receptor-mediated macrophage lipid accumulation and endothelial dysfunction in vitro (31-33).

\section{Oxidative Stress}

The vascular endothelium is an active organ, synthesizing and responding to vasodilator and vasoconstricting agents, regulating the balance between thrombosis and fibrinolysis, and interacting with monocytes and inhibiting platelet activity. Production of the inorganic gas nitric oxide (NO) is believed to be integral to many of these functions. NO, formed from L-arginine by nitric oxide syntheses, inhibits platelet aggregation and adhesion, modulates smooth muscle cell proliferation, attenuates the generation of endothelium, and reduces leukocyte adhesion to the endothelium. Dysfunction of the endothelium promotes thrombosis, inhibits fibrinolysis, recruits macrophages into the vascular wall by adhesion molecule expression and impairs vasodilation in response to appropriate stimuli. Hyercholesterolemia has repeatedly been shown to be associated with endothelial dysfunction, whereas cholesterol-lowering therapy has been demonstrated to result in improved endothelial dependent vasomotion. No studies have examined whether a reduction specifically in the circulating concentrations of triglyceride-rich lipoproteins result in an improvement in 
endothelial function. There are abundant potential mechanism by which endothelial dysfunction and atherogenic lipoproteins may be causally related. A study by Inoue et al. (34) reports an association between high serum remnant lipoprotein cholesterol and coronary vascular endothelial cell dysfunction and suggests that the cholesterol concentration in remnant lipoproteins may be taken as a marker of early stage coronary artery atherosclerosis that is not be detectable by angiogram. Available evidence suggests that triglyceride-rich lipoproteins and remnant particles increase the oxidative burden of the endothelium. The precise mechanisms whereby oxidative stress is increased, and locations of superoxide generation are controversial.

\section{Direct endothelial activity}

Triglyceride-rich lipoproteins and particularly their postprandial remnants have repeatedly been shown to be cytotoxic to cultured endothelial cells. There is also recent evidence to suggest that triglyceride-rich lipoproteins and their cholesterol-enriched remnants can cross the endothelial barrier and enter the arterial wall (27), placing them in a position where they may further promote endothelial damage. These particles can enter cells by means other than the LDL receptor (35) and do not require oxidation to increase cellular cholesteryl ester mass, in part because of their slow catabolism (36). Although the large size of chylomicrons and large VLDL might initially inhibit their entry into arterial intima, they may gain entry following size reduction on the surface of endothelial cells as a result of lipoprotein lipase (LPL) or endothelial lipase $(E L)$ induced hydrolysis of their core lipids. Once in the intima, their apo E content allows their uptake by macrophages by both LDL receptor and non-LDL receptor pathways (35).

\section{Prothrombotic changes}

A number of abnormalities of the thrombotic system have been associated with postprandial lipoproteins (37). Triglyceride-rich lipoproteins and their remnants have been shown to stimulate endothelial cell PAI-1 production (38). Elevated PAI-1 levels may be a marker of endothelial dysfunction, and cause a profound depression of fibrinolytic activity thus contributing to the pathogenesis of vascular disease. Lipolysed remnants of triglyceride-rich lipoproteins also promote an enhanced thrombogenic tendency by increasing circulating factor VII levels (39). A study by Orth, Luley and Wieland (40) demonstrated that postprandial chylomicrons and VLDL inhibit the ability of platelets to aggregate, whereas these effects were not observed for chylomicron remnants in physiological concentrations. Thus the authors conclude that the acute onset of myocardial infarction is not triggered by triglyceride-rich lipoproteins.

The triglyceride-rich lipoproteins with these athero- and thrombogenic cellular effects include plasma chylomicrons from normal as well as hyperlipoproteinemic subjects, and VLDL from subjects with elevated plasma triglycerides (29). It should be noted that these lipoproteins fractions contain remnants.

\section{Proinflammatory activity}

IDL, VLDL and postprandial remnant particles have been shown to be able to enter the artery wall and contribute to the lipid accumulation in lesions $(41,42)$, although in one of the studies there was no apoB ${ }_{48}$. found in the lesions. A recent study by Lee et al. (43) found that all Apo-B containing lipoproteins acquire proinflammatory activity when minimally oxidized and increase monocyte chemotaxis and adhesion. Their study indicates that the susceptibility of these lipoproteins to develop pro-inflammatory acivity is in part related to their fatty acid content, namely the ratio of $18: 1$ to $18: 2$ fatty acids. VLDL and chylomicrons as well as their remnants may be an important source of poly-unsaturated fatty acid containing phospholipids, and it is conceivable that their oxidation may generate substantial bioactivity (43). The lipid composition of lipoproteins can vary significantly depending on the individual diet. Oxidized lipids in lipoproteins can originate from two sources; either fatty acids are oxidized or the oxidized lipids were taken up with the food and were incorporated into chylomicrons (44) and may contribute to atherosclerosis lesion formation (45). Besides fatty acid composition the pro-inflammatory activity of chylomicrons also depends on the content of plateletactivating factor acetylhydrolase (PAF-AH) activity and the availability of antioxidants like vitamin E. Lee et al. (43) reported that although chylomicrons were relatively enriched in 18:1 fatty acids, which would make them less easily oxidized and thus less readily generate chemotactic activity they contained reduced levels of vitamin E per particle and no measurable PAF-AH activity. The authors conclude, that the absence of vitamin E and PAF-AH may make chylomicrons more susceptible to oxidation and might permit chylomicrons or their remnants more readily develop bioactivity when they are exposed to mildly oxidizing agents.

\section{Increase of endothelial expression of cell adhesion molecules}

The earliest observable abnormality of the vessel wall in animal models of atherosclerosis is enhanced monocyte adherence to the endothelium. This is mediated by alterations in the expression and activity of adhesion molecules and chemotactic proteins (46). Leukocyte and vascular cell adhesion molecules (CAMs) such as selectins, integrins, VCAM-1 and ICAM-1 play critical roles in the adhesion of monocytes to endothelial cells. The expression of E-selectin, ICAM-1 and VCAM- 1 is relatively low in normal vascular cells and is upregulated 
in response to various stimuli, including cytokines and oxidants, thus enabling monocytes to adhere to the vessel wall. In vivo animal studies and immunohistochemical studies of human tissues have shown that these CAMs are expressed at increased levels in atherosclerotic plaques (47). The normal endothelium is characterized by the production of a number of molecules that affect the contractile state of adjacent myocytes and the behavior of formed elements within the blood stream, and by the absence of cell surface adhesion molecules. Leukocyte adherence to endothelial cells required the surface expression of a number of adhesion molecules. In addition to stimulation by a number of cytokines, both cell adhesion and the expression of adhesion molecules are affected by lipoproteins. Triglyceride rich lipoproteins have a pro-adhesive effect, while HDL and apo-A1 inhibit the cytokine induced expression of adhesion molecules. Elevated levels of soluble adhesion molecules have been found in hyperlipidemia and coronary heart disease although their prognostic significance is not yet established (48). Leukocyte adherence to, and penetration of the endothelium are normal responses to tissue injury and can be induced by a number of cytokines such as TNF $\alpha$ and IL-1. The process starts with leukocyte rolling with subsequent adherence and finally migration (46). The rolling and adherent phases result from specific interactions between leukocytes and adhesion molecules expressed on endothelial cells. The initial rolling represents an interaction between leukocytes and selectins with subsequent adherence occurring through the involvement of ICAM and VCAM. Endothelial cells do not normally express these adhesion molecules on their luminal surfaces. In response to hypercholesterolemia, however, increases in the expression of molecules that mediate rolling and adhesion on the luminal surface of the endothelial cells occurs. Although soluble adhesion molecule concentrations are elevated in patients with hyperlipidemia and coronary heart disease, it is not clear whether the elevation is a consequence of the atherosclerotic process or is indeed an indication that adhesion molecule expression is an early and pivotal factor in disease development (48). Disorders in triglyceride metabolism may promote atherogenesis by increasing expression of vascular CAMs. Patients with markedly elevated triglyceride levels also have decreased HDL levels and adnormalities in fatty acid metabolism. In vitro studies have shown that low HDL and oxidized fatty acids increase the endothelial expression of CAMs in response to cytokines. CAMs are also present in the circulation as soluble forms, which lack the membrane-spanning and cytoplasmic domains that are present in the membrane-bound forms. Although the origins, metabolism, and functional significance of CAMs are not fully understood, the quantitative assessment of the levels of CAMs is straightforward. These levels have been noted to be elevated in certain pathological conditions such as sepsis and autoimmune diseases in which tissue expression of the membrane-bound forms of CAMs is also known to be upregulated. In one study (47) levels of E-Selectin, ICAM-1 and VCAM-1 in the serum of patients with hypertriglyceridemia were increased, and multivariant analyses showed, that this increase was independent of other risk factors. The lysophosphatidylcholine component of oxidized lipoproteins appears to be responsible for the selective induction of VCAM-1 and ICAM-1 expression in arterial endothelial cells; it is also a chemoattractant for mononuclear cells (49). Experiments in our laboratory have expanded this concept. In unpublished experiments we have assessed the ability of triglyceride- rich lipoprotein fractions from normal and diabetic patients to increase the ability of endothelial cells to bind monocytes. Normal individuals and diabetics were studied. In some but not all individuals triglyceride-rich lipoprotein gathered during the postprandial period were more effective at inducing endothelial cells than the same fractions before the meal. The best predictor of the degree to which a fraction would be able to induce adhesion was its triacylglycerol and lysolecithin content. This is consistent with the hypothesis that individuals with delayed remnant removal accumulate lipoproteins that may have a role in inducing early atherosclerotic lesions during the postprandial period. Consonant with this Carantoni et al. noted that mononuclear cells from diabetics were more likely to adhere to endothelial cells than those from controls (50).

\section{Interaction with circulating blood cells}

The cell types that are involved in atherosclerosis include circulating blood cells, especially monocytes. These are exposed to the highest levels of circulating chylomicrons and remnants and are the precursors of foam cells. Several studies have been published that show that mononuclear cells isolated from patients with hypercholesterolemia or hyper-triglyceridemia or both, such as occurs often in diabetics, have an increased adherence to the endothelium (51-53).

\section{Indirect involvement of chylomicron remnants in atherogenesis}

In addition to the ways in which remnants might directly affect specific steps in atherogenesis as discussed above, it is possible that they expand this effect by altering the metabolism of the other lipoprotein classes. There is relatively little evidence for this. Delayed remnant removal is part of the metabolic syndrome which, when fully expressed, is characterized by insulin resistance, hypertension, increased fasting triglycerides, small dense LDL and low HDL. Whether delayed remnant removal is the cause of any of these abnormalities or is one of the consequences of the underlying defects is unknown since the causative defects have yet to be fully elucidated. The 
association of delayed remnant removal with other lipoprotein abnormalities has been commented on frequently.

\section{Effect on LDL particles}

Triglyceride-rich lipoproteins may also influence endothelial function indirectly, by shifting the distribution of the circulating LDL population towards smaller, denser species. Plasma triglyceride is known to be an important factor associated with reduced LDL size. Recent evidence suggests that this relationship exhibits threshold behavior. In a recent study of patients with type 2 diabetes, LDL size was related to endothelium dependent vasodilation even after controlling for age, glycated haemoglobin, blood pressure and cholesterol (54).

\section{Effect on HDL particles}

Metabolism of HDL lipoproteins and triglyceride-rich particles are closely related as reflected by the inverse relationship between plasma triglyceride and plasma HDL cholesterol levels. Lipids from HDL particles and triglyceride-rich lipoproteins are in constant exchange. Cholesterylesters are transferred from HDL to triglyceride-rich particles in exchange for triglycerides by the cholesterol-ester-transfer protein. Over time, this produces triglyceride-enriched HDL particles that are a better substrate for the enzyme hepatic lipase (HL). HL removes triglyceride from the HDL particles, which results in smaller HDL particles that are metabolized faster than larger HDL particles. This process leads to lower HDLcholesterol levels. HDL-cholesterol level was found to be related to endothelium-dependent relaxation and a study by Chowienczyk (55) suggests that the higher HDLcholesterol concentrations in women may be responsible for their observed protection against the adverse effects of hypercholesterolemia on endothelium-dependent vasodilatation.

\section{Conclusion and Hypothesis}

Epidemiologic studies have provided strong support for the association between delayed remnant removal and premature atherosclerosis. This seems to be especially applicable to patients with normal LDL levels and with some of the symptoms of the metabolic syndrome such as high triglycerides, and insulin resistance. Remnants have been found to affect several processes that are currently believed to play a role in atherogenesis. Specifically they may induce monocyte binding to endothelial cells, and macrophages to become enriched with lipids and form foam cells. It is suspected that the effects on endothelial cells are mediated by lysolecithin found during the lipolysis of chylomicrons as well as by oxidized lipids derived from the diet. It is our hypothesis that in individuals with delayed removal of chylomicron remnants, the prolonged exposure of areas of endothelium, that have been partially activated by turbulent flow, to specific components of the remnants, results in the endothelial cells becoming further activated and able to bind monocytes. During or shortly after the transcytosis to the intima and transformation of monocytes to macrophages, the macrophages become engorged with remnant derived lipids and form the nidus of a fatty streak.

\section{References}

(1) Patsch JR, Miesenbock G, Hopferwieser T, Muhlberger V, Knapp E, Dunn JK, Gotto AM Jr, and Patsch W: Relation of triglyceride metabolism and coronary artery disease. Studies in the postprandial state. Arterioscler Thromb, 12: 1336-1345, 1992

(2) Zilversmit DB: Atherogenesis: A postprandial phenomenon. Circulation, 60: 473-485, 1979

(3) Hussain MM, Kancha RK, Zhou Z, Luchoomun J, $\mathrm{Zu} \mathrm{H}$, and Bakillah A: Chylomicron-chylomicron remnant clearance by liver and bone marrow in rabbits. Factors that modify tissue-specific uptake. J Biol Chem, 264: 9571-9582, 1989

(4) Hussain MM, Kahley RW, Boyles JK, Fainaru M, Brecht WJ, and Lindquist PA: Chylomicron assembly and catabolism: role of apolipoproteins and receptors. Biochim Biophys Acta, 1300: 151-170, 1996

( 5 ) Karpe F, Olivecrona T, Hamsten A, and Hultin M: Chylomicron/chylomicron remnants turnover in humans: evidence for margination of chylomicrons and prior conversion of larger to smaller chylomicron remnants. J Lipid Res, 38: 949-961, 1997

( 6 ) Beisiegel U: Lipoprotein metabolism. Eur Heart J, 19 (Suppl) A: A20-A23, 1998

(7) Summary of the second report of the National Cholesterol Education Program (NCEP) Expert Panel on Detection, Evaluation, and Treatment of High Blood Cholesterol in Adults (Adult Treatment Panel II. Jama, 269: 3015-3023, 1993

( 8 ) Libby P and Aikawa M: Stabilization of atherosclerotic plaques: New mechanisms and clinical targets. Nat Med, 8: 1257-1262, 2002

( 9 ) Ross R: Atherosclerosis-an inflammatory disease. N Engl J Med, 340: 115-126, 1999

(10) Ross R and Glomset JA: The pathogenesis of atherosclerosis. I. N Engl J Med, 295: 369-377, 1976

(11) Ross R and Glomset JA: The pathogenesis of atherosclerosis. II. N Engl J Med, 295: 420-425, 1976

(12) Williams KJ and Tabas I: The response-to-retention hypothesis of early atherogenesis. Arterioscler Thromb Vasc Biol, 15: 551-561, 1995 
(13) Glagov S, Weisenberg E, Zarins CK, Stankunavicius $\mathrm{R}$, and Kolettis GJ: Compensatory enlargement of human atherosclerotic coronary arteries. N Engl J Med, 316: 1371-1375, 1987

(14) Jonasson L, Holm J, Skalli O, Bondjers G, and Hansson GK: Regional accumulations of $T$ cells, macrophages, and smooth muscle cells in the human atherosclerotic plaque. Arteriosclerosis, 6 : 131-138, 1986

(15) Van der Wal AC, Das PK, Bentz van de Berg D, van der Loos CM, Becker AE: Atherosclerotic lesions in humans. In situ immunophenotypic analysis suggesting an immune mediated response. Lab Invest, 61: 166-170, 1989

(16) Libby R and Ross R: Cytokines and growth regulatory molecules. In: Atherosclerosis and Coronary Artery Disease. ed by Fuster V, Ross R, and Topol EJ, pp 585-594, Lipincott, Philadelphia, 1996

(17) Rains EW and Ross R: The role of macrophages. In: Atherosclerosis and Coronary Artery Disease. ed by Fuster V, Ross R, and Topol EJ, pp 539-555, Lipincott, Philadelphia, 1996

(18) Falk E, PKS, and VF: Pathogenesis of plaque distruption. In: Atherosclerosis and Coronary Artery Disease. ed by Fuster V, Ross R, and Topol EJ, pp 539-555, Lipincott, Philadelphia, 1996

(19) Stender S and Christensen S: The concomitantly measured transfer of free cholesterol, esterified cholesterol, phospholipids and phosphoprotein from plasma into the aortic wall of stilboesteroltreated cockerels. Atherosclerosis, 28: 15-28, 1977

(20) Stender S: Arterial entrance and metabolism of free and esterified plasma cholesterol measured in vivo in experimental animals by a dual isotope method. Atherosclerosis, 32: 1129-1139, 1979

(21) Nordestgaard BG, Tybjaerg-Hansen A, and Lewis B: Influx in vivo of low density, intermediate density, and very low density lipoproteins into aortic intimas of genetically hyperlipidemic rabbits. Roles of plasma concentrations, extent of aortic lesion, and lipoprotein particle size as determinants. Arterioscler Thromb, 12: 6-18, 1992

(22) Fry DL, Haupt MW, and Pap JM: Effect of endothelial integrity, transmural pressure, and time on the intimal-medial uptake of serum 125l-albumin and $125 \mathrm{I}-\mathrm{LDL}$ in an in vitro porcine arterial organsupport system. Arterioscler Thromb, 12: 13131328, 1992

(23) Nordestgaard BG and Tybjaerg-Hansen A: IDL, VLDL, chylomicrons and atherosclerosis. Eur $\mathrm{J}$ Epidemiol, 8 (suppl.1): 92-98, 1992

(24) Nordestgaard BG and Nielsen LB: Atherosclerosis and arterial influx of lipoproteins. Curr Opin Lipidol, 5: 252-257, 1994

(25) Nordestgaard BG: The vascular endothelial barrier-selective retention of lipoproteins. Curr Opin Lipidol, 7: 269-273, 1996

(26) Nordestgaard BG, Stender S, and Kjeldsen K: Reduced atherogenesis in cholesterol-fed diabetic rabbits. Giant lipoproteins do not enter the arterial wall. Arteriosclerosis, 8: 421-428, 1988

(27) Mamo JC and Wheeler JR: Chylomicrons or their remnants penetrate rabbit thoracic aorta as efficiently as do smaller macromolecules, including low-density lipoprotein, high-density lipoprotein, and albumin. Coron Artery Dis, 5: 695-705, 1994

(28) Proctor SD and Mamo JC: Arterial fatty lesions have increased uptake of chylomicron remnants but not low-density lipoproteins. Coron Artery Dis, 7: 239245, 1996

(29) Gianturco SH and Bradley WA: Pathophysiology of triglyceride-rich lipoproteins in atherothrombosis: cellular aspects. Clin Cardiol, 22 (6 Suppl): II7-II14, 1999

(30) Steiner G: Intermediate-density lipoproteins, diabetes and coronary artery disease. Diabetes Res Clin Pract, 40 (Suppl): S29-33, 1998

(31) Gianturco SH, Eskin SG, Navarro LT, Lahart CJ, Smith LC, and Gotto AM, Jr: Abnormal effects of hypertriacylglycerolemic very low-density lipoproteins on 3-hydroxy-3-methylglutaryl coenzyme a reductase activity and viability of cultured bovine aortic endothellal cells. Biochim Biophys Acta, 618 : 143-152, 1980

(32) Li, X, N, Koons JC, Benza RL, Parks JM, Varma VK, Bradley WA, Gianturco SH, Taylor KB, Grammer JR, Tabengwa EM, and Booyse FM: Hypertriglyceridemic VLDL decreases plasminogen binding to endothelial cells and surface-localized fibrinolysis. Biochemistry, 35: 6080-6088, 1996

(33) Li, X, N, Grenett HE, Benza RL, Demissie S, Brown SL, and Tabengwa EM Gianturco SH, Bradley WA, Fless GM, and Booyse FM: Genotype-specific transcriptional regulation of $\mathrm{PAl}-1$ expression by hypertriglyceridemic VLDL and $L p(a)$ in cultured human endothelial cells. Arterioscler Thromb Vasc Biol, 17: 3215-3223, 1997

(34) Inoue T, Saniabadi AR, Matsunaga R, Hoshi K, Yaguchi,I and Morooka S: Impaired endotheliumdependent acetylcholine-induced coronary artery relaxation in patients with high serum remnant lipoprotein particles. Atherosclerosis, 139: 363-367, 1998

(35) Fujioka, Y., A.D. Cooper, and L.G. Fong: Multiple processes are involved in the uptake of chylomicron remnants by mouse peritoneal macrophages. J Lipid Res, 39: 2339-2349, 1998

(36) Ellsworth JL, Fong LG, Kraemer FB, and Cooper $A D$ : differences in the processing of chylomicron remnants and beta-VLDL by macrophages. J Lipid 
Res, 31: 1399-1411, 1990

(37) Roche, H.M. and M.J. Gibney: The impact of postprandial lipemia in accelerating atherothrombosis. J Cardiovasc Risk, 7: 317-324, 2000

(38) Eriksson P, Nilsson L, Karpe F, and Hamstem A: Very-low-density lipoprotein response element in the promoter region of the human plasminogen activator inhibitor-1 gene implicated in the impaired fibrinolysis of hypertriglyceridemia. Arterioscler Thromb Vasc Biol, 18: 20-26, 1998

(39) Saigo M, Abe S, Ogawa M, Biro S, Minagoe S, Maruyama I, Toda H, Kiyonaga K, Atsuchi Y, Tahara $\mathrm{M}$, Mawatari K, and Tei C: Plasma level of triglyceride-rich lipoprotein remnants is closely associated with the activation of coagulation factor VII in patients with myocardial infarction. Thromb Res, 100: 9-17, 2000

(40) Orth, M., C. Luley, and H. Wieland: Effects of VLDL, chylomicrons, and chylomicron remnants on platelet aggregability. Thromb res, 79: 297-305, 1995

(41) Rapp JH, ALespine A, Hamilton RL, Colyvas N, Chaumeton AH, Tweedie-Hardman J, Kotite L, Kunitake ST, Havel RJ, and Kane JP: Triglyceriderich lipoproteins isolated by selected-affinity antiapolipoprotein B immunosorption from human atherosclerotic plaque. Arterioscler Thromb, 14: 1767-1774, 1994

(42) Chung BH, Tallis G, Yalamoori V, Anantharamaiah GM, and Segrest JP: Liposome-like particles isolated from human atherosclerotic plaques are structurally and compositionally similar to surface remnants of triglyceride-rich lipoproteins. Arterioscler Thromb, 14: 622-635, 1994

(43) Lee C, Sigari F, Segrado T, Horkko S, Hama S, Subbaiah PV, Miwa M, Navab M, Witztum JL, and Reaven PD: All ApoB-containing lipoproteins induce monocyte chemotaxis and adhesion when minimally modified. Modulation of lipoprotein bioactivity by platelet-activating factor acetylhydrolase. Arterioscler Thromb Vasc Biol, 19: 1437-1446, 1999

(44) Staprans I, Rapp LH, Pan XM, Kim KY, and Feingold KR: Oxidized lipids in the diet are a source of oxidized lipid in chylomicrons of human serum. Arterioscler Thromb, 14: 1900-1905, 1994

(45) Staprans I, Pan XM, Rapp JH, and Feingold KR: Oxidized cholesterol in the diet accelerates the development of aortic atherosclerosis in cholesterolfed rabbits. Arterioscler Thromb Vasc Biol, 18: 977-
983, 1998

(46) Butcher EC: Leukocyte-endothelial cell recognition: Three (or more) steps to specificity and diversity. Cell, 67: 1033-1036, 1991

(47) Abe Y, El-Masri B, Kimball KT, Pownall H, Reilly $\mathrm{CF}$, Osmundsen K, Smith CW, and Ballantyne CM: Soluble cell adhesion molecules in hypertriglyceridemia and potential significance on monocyte adhesion. Arterioscler Thromb Vasc Biol, 18: 723-731, 1998

(48) Dart, A.M. and J.P. Chin-Dusting: Lipids and the endothelium. Cardiovasc Res, 43: 308-322, 1999

(49) Kume N, Cybulsky MI, and Gimbrone MA: Lysophosphatidylcholine, a component of atherogenic lipoproteins, induces mononuclear leukocyte adhesion molecules in cultured human and rabbit arterial endothelial cells. J Clin Invest, 90: 11381144, 1992

(50) Carantori M: Adherence of mononuclear cells to endothelium in vitro is increased in patients with NIDDM. Diabetes Care, 20: 1462-1465, 1997

(51) De Gruijter M, Hoogerbrugge N, van Rijn MA, Koster JF, Sluiter W, and Jongkind JF: Patients with combined hypercholesterolemia-hypertriglyceridemia show an increased monocyte-endothelial cell adhesion in vitro: triglyceride level as a major determinant. Metabolism, 40: 1119-1121, 1991

(52) Jongkind JF, Verkerk A, and Hoogerbrugge N: Monocytes from patients with combined hypercholesterolemia-hypertriglyceridemia and isolated hypercholesterolemia show an increased adhesion to endothelial cells in vitro: II. Influence of intrinsic and extrinsic factors on monocyte binding. Metabolism, 44: 374-378, 1995

(53) Hoogerbrugge N, Verkerk A, Jacobs ML, Postema PT, and Jongkind JF: Hypertriglyceridemia enhances monocyte binding to endothelial cells in NIDDM. Diabetes Care, 19: 1122-1125, 1996

(54) Makimattila S, Liu ML, Vakkilainen J, Schlenzka A, Lahdenpera S, Syvanne M, Mantysaari M, Summanen P, Bergholm R, Taskinen MR, and Yki-Jarvinen $\mathrm{H}$ : Impaired endothelium-dependent vasodilation in type 2 diabetes. Relation to LDL size, oxidized LDL, and antioxidants. Diabetes Care, 22: 973-981, 1999

(55) Chowienczyk PJ, Watts GF, Cockcroft JR, Brett SE, and Ritter JM: Sex differences in endothelial function in normal and hypercholesterolaemic subjects. Lancet, 344: 305-306, 1994 